\title{
Detection of Drilled Hole on Subsurface of Aluminum Plate with Rayleigh Ultrasonic Wave Field by Laser Probing
}

\author{
Kazuhiko Imano* \\ Graduate School of Science and Engineering, Akita University, \\ 1-1 Tegata Gakuen-cho, Akita City, 010-8502 Japan
}

(Received October 23, 2019; accepted January 23, 2020)

Keywords: laser probe method, Rayleigh wave, phase change, A0 mode Lamb wave, mode conversion, FEM analysis

A drilled hole with a diameter of less than or equal to the wavelength of a surface acoustic wave on the subsurface of an aluminum plate is detected by laser probing. The holes that could not be detected by a conventional method are detected from the phase change of the Rayleigh wave included in the reflected laser light. Experiments involving $5 \mathrm{MHz}$ Rayleigh waves propagating along the surface of an aluminum plate are performed by laser probing. Finite element method (FEM) analysis reveals mode conversion from a Rayleigh wave to an A0 mode Lamb wave between the drilled hole and the material surface. The phase change at the edge of the drilled hole that causes the difference in sound velocity between the Rayleigh and Lamb waves is also described.

\section{Introduction}

Ultrasonic waves have widely been used in the nondestructive evaluation (NDE) of pipes and plates. For detecting defects such as delaminations, voids, and holes, a pulse-echo method has been employed. ${ }^{(1,2)}$ However, the detection of defects, such as defects, compared with ultrasound wavelength, may not be easy via a conventional pulse-echo method. This is because the detection surface of the ultrasonic probe has a finite size, and if it is much larger than the defect, defect information is spatially averaged on the probe surface. Therefore, an efficient method of acquiring information on very small defects has been desired. On the other hand, ultrasonic measurement using a laser has been widely performed. ${ }^{(3-8)}$ It is used to study defects and other physical properties, such as cracks in materials, using the interaction between laser light and ultrasonic waves, and to inspect composite materials using Lamb waves. ${ }^{(9-11)}$ Laser light is concentrated in a small area using an optical fiber, ${ }^{(12,13)}$ and it is used as a sound source or a sensor such as Lamb waves. In many cases, the surface of the target is often measured, ${ }^{(14)}$ but there are not many studies on the internal physical properties of a solid material, such as defects under the subsurface of an object.

*Corresponding author: e-mail: imano@gipc.akita-u.ac.jp

https://doi.org/10.18494/SAM.2020.2659 
In this study, the laser light reflected from the surface of a metal plate sample is used as a probe to acquire phase information, which is phase-modulated by an ultrasonic wave. Drilled holes under the subsurface of a sample also affect the phase of an ultrasonic wave. Thus, the phase of the reflected light from the sample surface is also modulated by an ultrasonic wave and a defect. When the excitation signal is known and can be controlled arbitrarily, the measured phase containing a defect can be extracted from an unusual phase change. This phase difference can be picked up by optical methods such as the use of laser beam light. This method is called 'laser probing', which has already been used to measure residual stress in glass and plastic materials. ${ }^{(2,3-17)}$ This method enables us to observe the characteristics of a smaller area than other imaging methods, such as the photoelasticity method. In addition, in this method, contact with the sample is unnecessary and there is no problem of spatial signal averaging described above. Most conventional ultrasonic transducers are millimeter to several tens of $\mathrm{cm}$ in size, but with laser probing, a high spatial resolution of the order of micrometer size beam spot can easily be obtained. In the following, an example of detecting a drilled hole immediately below the surface of an aluminum plate sample is shown to clarify the efficiency of this method.

\section{Experimental System and Method}

\subsection{Figures and tables}

Figure 1 shows a reflection-type laser probing system. ${ }^{(17)} 30$ cycles of burst sine wave voltage signals are repeatedly generated by a function generator (KEYSIGHT: 33600A) with a period of $10 \mathrm{~ms}$ and fed to a bipolar amplifier (NF: HSA 4101) for amplification up to about $100 \mathrm{~V}$. The frequency of the burst sine wave is set to $5 \mathrm{MHz}$ to match the resonance frequency of the piezoelectric transducer. A Rayleigh wave probe (Japan probe: $5 Z 10 \times 10 \mathrm{R}$ ) is attached as a piezoelectric transmitter to the surface of an aluminum plate. A Rayleigh wave is a type of surface wave that propagates on the surface of the propagation medium used and is convenient for examining the characteristics of the sample surface. The hole to be inspected is $0.5 \mathrm{~mm}$ in diameter and $5 \mathrm{~mm}$ in depth and is drilled using a small end mill. A Rayleigh wave propagates above the hole about $0.3 \mathrm{~mm}$ below the sample surface. Then, a laser beam (He-Ne)

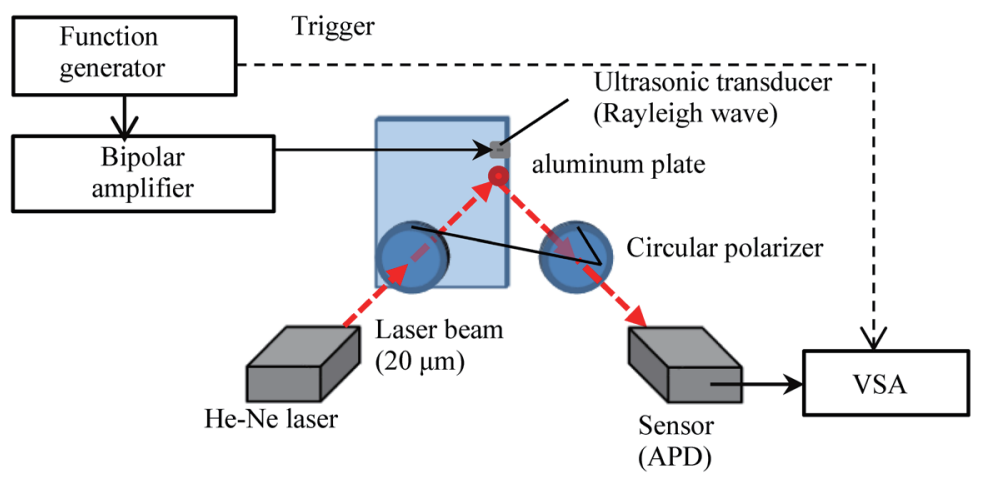

Fig. 1. (Color online) Experimental setup for laser probing to detect small defects. 
spot of about $20 \mu \mathrm{m}$ in diameter is irradiated via a polarizer (first polarized plate) to scan the measurement point.

As shown in Fig. 1, second circular polarizing plates (analyzer) are inserted between the sample surface and the optical sensor to receive only the polarized components reflected on the surface of the aluminum plate. Laser light is received by the avalanche photodiode optical sensor (APD: Hamamatsu Photonics C5460 module). ${ }^{(2,15-20)}$ The propagation medium is perturbed by the Rayleigh wave propagating on the sample surface and, as a result, the reflected laser light is phase-modulated by the Rayleigh wave that passes through the analyzer (second polarized plate) and is received by the APD. Thus, the reflected laser light contains information on the change in propagation mode caused by the Rayleigh wave. As shown in Fig. 1, the signal acquired from the APD is obtained as the time waveform of ultrasonic waves proportional to the sound pressure, ${ }^{(19,20)}$ and this signal is fed to the vector signal analyzer (VSA: Agilent 89441A). The input signal is subjected to quadrature synchronous detection, then the phase data against the propagation distance $\left(d \theta^{\prime} / d l\right.$ and $\left.d \theta / d l\right)$ are obtained from the ultrasonic waveform, where $l$ is the propagation distance. The phase change ratio $d \theta^{\prime} / d \theta$ is obtained from two phase data, where $d \theta^{\prime}$ and $d \theta$ are the phase changes with and without the hole, respectively.

Figure 2(a) shows the dimensions of the aluminum sample $\left(100 \times 100 \times 10 \mathrm{~mm}^{3}\right)$ used in the experiment. Figure 2(b) shows the enlarged view of the machined part. As mentioned above, the Rayleigh wave probe (piezotransducer size: $10 \times 10 \mathrm{~mm}^{2}$ ) is attached to the sample surface. The Rayleigh wave is measured for the phase against the wave propagation and used to examine the presence of holes in the sample.

In the experiment, laser light is irradiated at a position $4 \mathrm{~mm}$ away from the Rayleigh wave transducer to the back of the hole as shown in Fig. 2(b). In Fig. 2(b), the reflected laser light signal for a total of 4000 points is received by the APD at $1 \mu \mathrm{m}$ intervals in the measurement range of $4 \mathrm{~mm}(-3-+1 \mathrm{~mm})$. The ultrasonic time waveform data obtained from the APD are fed to the VSA and acquired as phase data of 4000 points. The phase data acquired above are averaged every $100 \mu \mathrm{m}$ (near the hole) or $250 \mu \mathrm{m}$ (other position) to obtain the regression lines with the phase slopes $d \theta^{\prime} / d l$ and $d \theta / d l$. Finally, average phase slopes were calculated. The phase change ratio $1 /\left(d \theta^{\prime} / d \theta\right)$ values of 16 points are plotted against the propagation distance $l(\mathrm{~mm})$.

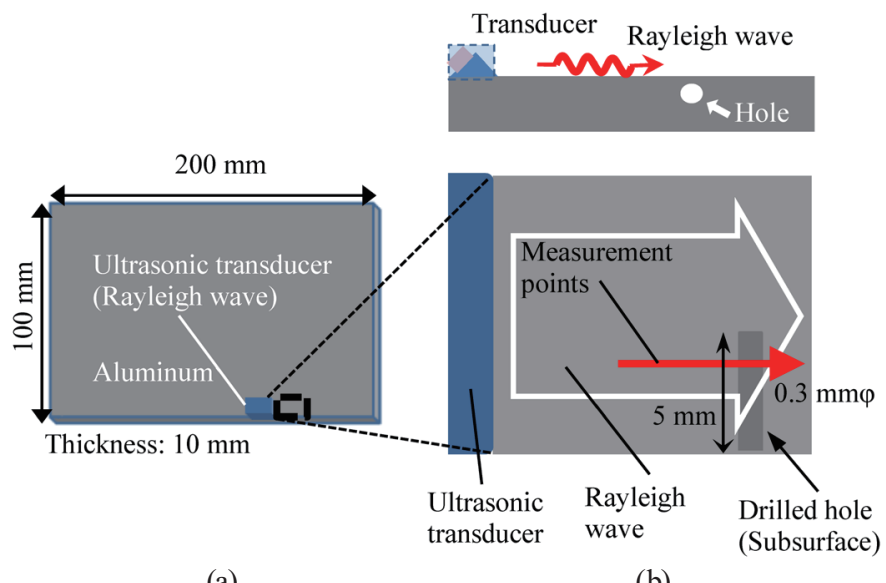

(a)

(b)

Fig. 2. (Color online) Schematics of (a) an aluminum plate (10 $\mathrm{mm}$ thick) with a small hole and (b) enlarged view of the machined part. 


\section{Experimental Results and Finite Element Method (FEM) Analysis}

\subsection{Phase characteristics over the hole in the sample}

Figure 3 shows the experimental results for the phase change ratio of the wave propagating on the surface of the sample measured from the reflected laser beam. As seen from the figure, the phase change is reversed front and back of the hole position. As described above, the difference in phase change ratio $1 /\left(d \theta^{\prime} / d \theta\right)$ obtained from the slope of each phase change with and without defects is clearly identified by this method.

\subsection{Results of FEM analysis}

Figures 4(a)-4(c) show the results of FEM analysis when the Rayleigh wave is generated in the sample by oblique incidence, which is the same condition as the experiments. In the absence of defects, as shown in Fig. 3(a), the Rayleigh wave propagates on the sample surface and the phase slope decreases uniformly. On the other hand, in Fig. 3(b), the Rayleigh wave propagation mode changes different from the mode around the hole position. Figure 3(c) shows an enlarged view around the hole. From Fig. 3(c), we observe that the Rayleigh wave begins mode conversion near the left side of the drilled hole.

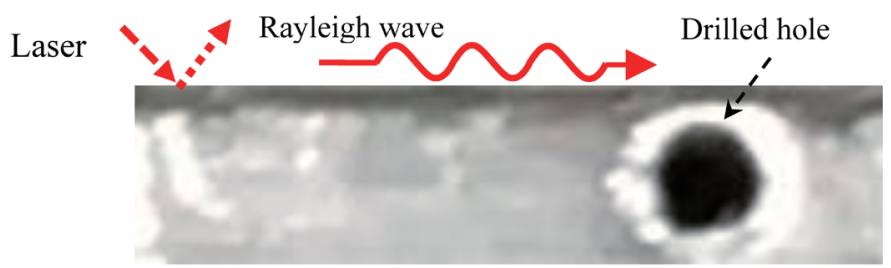

(a)

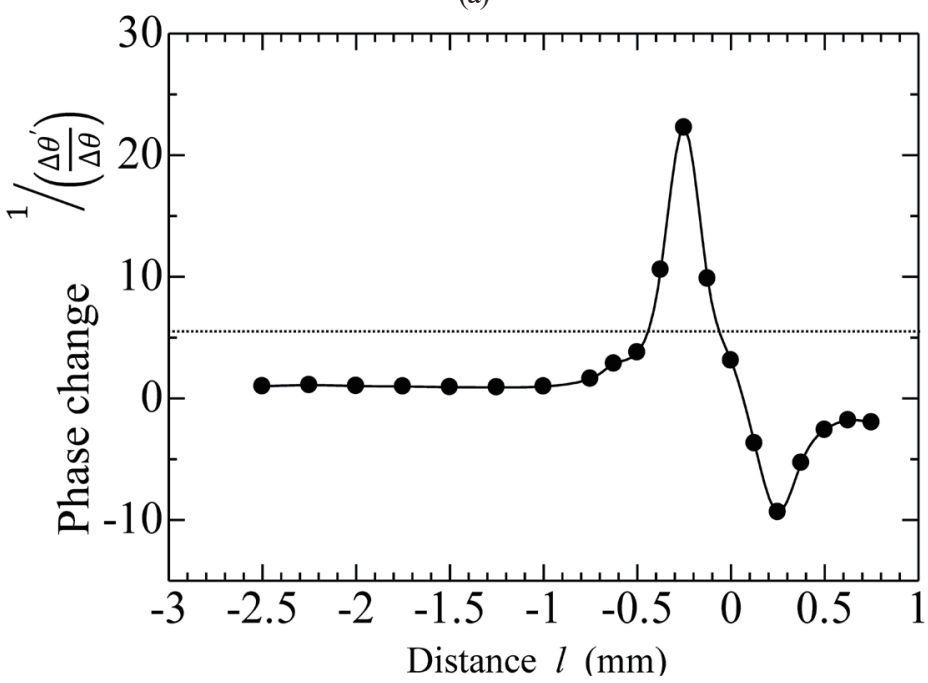

(b)

Fig. 3. (Color online) (a) Photograph of hole in aluminum plate and (b) phase change around the hole. $l=0$ indicates hole position. 

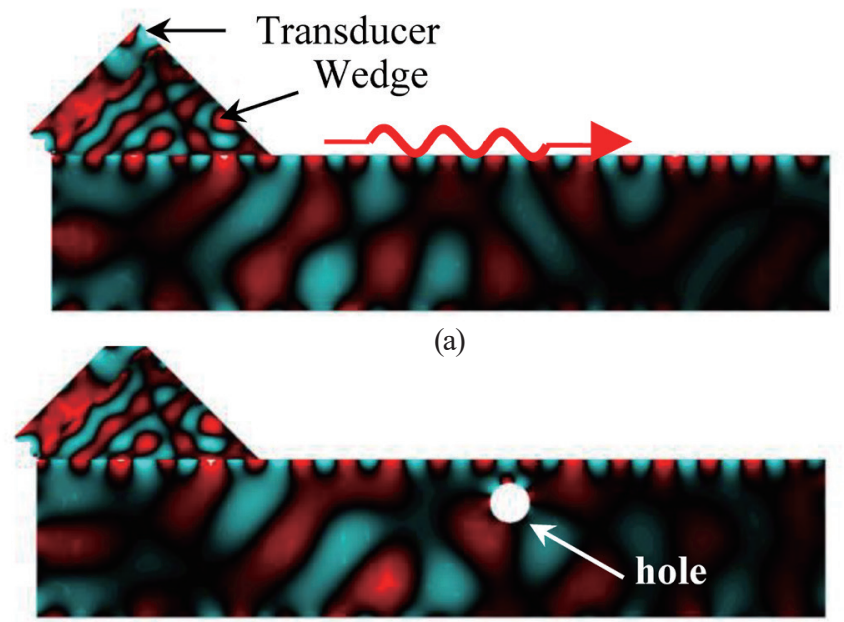

(b)

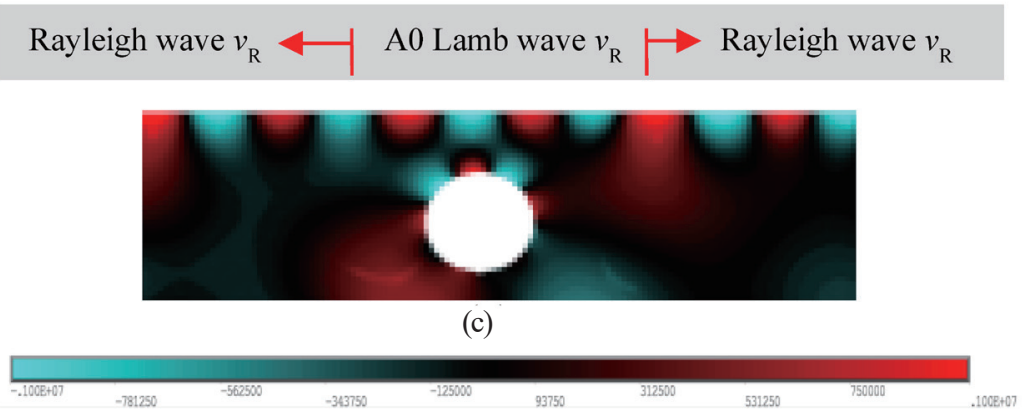

Fig. 4. (Color) FEM analysis of ultrasonic wave in sample. Rayleigh wave on sample surface (a) without and (b) with hole, and (c) enlarged view of Rayleigh and A0 mode Lamb waves.

\section{Discussion}

From the results in Figs. 3 and 4, we consider the cause of the phase change at the top of the circular defect. As shown in Fig. 4, the Rayleigh wave becomes the A0 mode Lamb wave between the hole and the sample surface after passing around the hole, and the A0 mode Lamb wave transforms into the Rayleigh wave again. That is, since sound velocity decreases (Rayleigh wave: $v_{R} \rightarrow A 0$ mode Lamb wave: $v_{L}$ ) at the front side of the hole, phase delay decreases. In contrast, sound velocity increases (A0 mode Lamb wave $\rightarrow$ Rayleigh wave) at the rear side of the hole, so the phase delay increases.

Figure 5 shows the calculated result of the dispersion curve of the A0 Lamb wave. Figure 5 indicates the phase velocity of the Rayleigh wave and the dispersion curve of the A0 mode Lamb wave. At the measurement frequency of $5 \mathrm{MHz}$, the Rayleigh wave velocity (about $90 \mathrm{~m} / \mathrm{s}$ ) is larger than the A0 mode Lamb wave velocity. This difference in velocity between both waves may cause the phase change around the hole. 


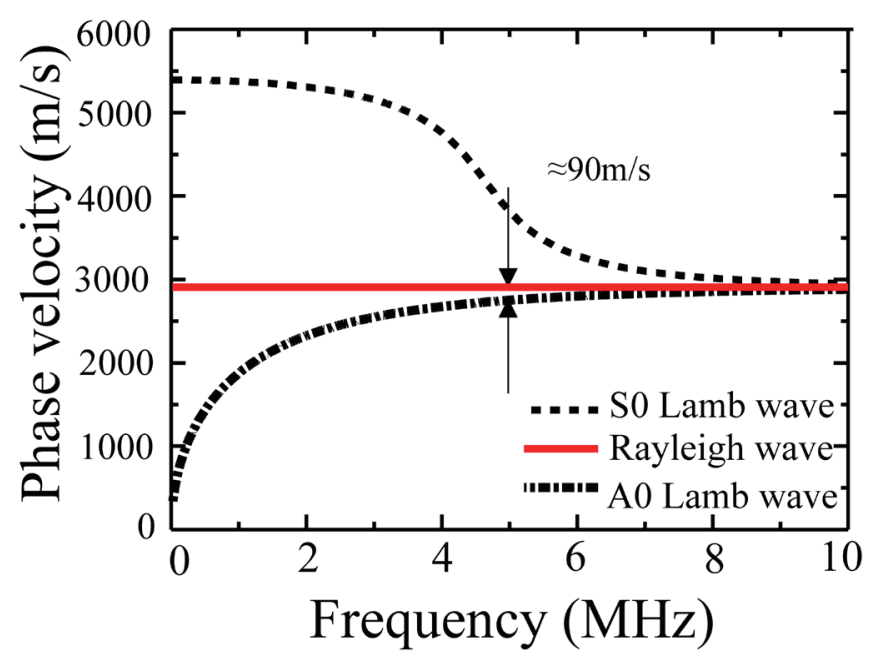

Fig. 5. (Color online) Dispersion curves of phase velocity for Rayleigh and A0 mode Lamb waves. At $5 \mathrm{MHz}$, the velocity difference of both waves is about $90 \mathrm{~m} / \mathrm{s}$.

\section{Conclusion}

A drilled hole with a diameter of less than or equal to the wavelength of a surface acoustic wave on the subsurface of an aluminum plate is detected by laser probing. The holes that could not be detected by a conventional method are detected from the phase change of the Rayleigh wave included in the reflected laser light. Experiments involving $5 \mathrm{MHz}$ Rayleigh wave propagation along the surface of an aluminum plate are performed by laser probing. FEM analysis reveals mode conversion from a Rayleigh wave to an A0 mode Lamb wave between the drilled hole and the material surface. The phase change at the edge of the drilled hole that causes the difference in sound velocity between the Rayleigh and Lambs waves is also confirmed. Using our method, information on depths of various defects, such as delaminations, cracks, and so forth, can be used for the detection of holes by changing the Rayleigh wave frequency for various defects. The dependence on the size (diameter/depth), position, and number of holes is important and should be examined under various conditions. Future work of this method will be performed to examine the measurement accuracy and applied to the NDE of defects near the surface of a material.

\section{Acknowledgments}

This work was supported by JSPS KAKENHI Grant Number JP19K04404.

\section{References}

1 W. S. Lester: Fundamentals of Ultrasonic Nondestructive Evaluation: A Modeling Approach (Springer AG, Switzerland, 2016) 2nd. ed., p. 13.

2 K. Imano: Jpn. J. Opt. 44 (2015) 488 (in Japanese). 
3 M. Ševčika, T. Grabec, P. Stoklasova, M. Janovska, K. Zoubkova, P. Sedlak, H. Seiner, and M. Landa: Acta. Phys. Pol. A 134 (2018) 807.

4 J. A. Guggenheim, E. Z. Zhang, and P. C. Beard: IEEE Trans. UFFC 64 (2017) 1857.

5 H. Isobe and K. Hara: Proc. 9th Int. Conf. (JSME, 2017) 068.

6 A. A. Karabutov, N. B. Podymova, and E. B. Cherepetskaya: J. Appl. Mech. Tech. Phys. 58 (2017) 503.

7 J.-Y. Park and J.-R. Lee: J. Mech. Sci. Tech. 31 (2017) 4099.

8 Z. Tian, S. Howden, W. Ma, Z. W. Xiao, and L. Yu: Mech. Syst. Sig. Process. 121 (2018) 158.

9 F. Gao, J. Hua, L. Zeng, and J. Lin: Ultrasonics 93 (2019) 122.

10 M. Faisal Haider, M. Y. Bhuiyan, B. Poddar, B. Lin, and V. Giurgiutiu: J. Sound Vib. 431 (2018) 212.

11 C. Tao, H. Ji, J. Qiu, C. Zhang, Z. Wang, and W. Yao: Compos. Struct. 166 (2017) 219.

12 X. Yin, Y. Shen, D. Su, and Z. Shao: Opt. Commun. 453 (2019) 124422.

13 A. A. Karabutov and Y. G. Sokolovskaya: Moscow Univ. Phys. Bull. 73 (2018) 622.

14 X. Liu, S. Yang, Y. Liu, Y. Chi, and X. Gu: Appl. Sci. (Switzerland) 8 (2018) 1796.

15 K. Imano and M. Akatsuka: Int. J. Soc. Eng. Resour. 30 (2019) 1 (in Japanese).

16 M. Akatsuka and K. Imano: Int. J. Soc. Eng. Resour. 30 (2019) 13 (in Japanese).

17 M. Akatsuka and K. Imano: Int. J. Soc. Eng. Resour. 30 (2019) 18 (in Japanese).

18 K. Imano: Int. J. Soc. Eng. Resour. 27 (2016) 6 (in Japanese).

19 T. Okoda and Y. Sononda Jpn. J. Appl. Phys. 42 (2003) 5821.

20 Y. Sonoda: J. Acoust. Soc. Jpn. 62 (2006) 571 (in Japanese).

\section{About the Author}

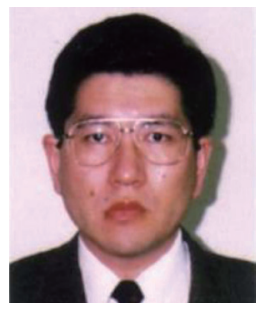

Kazuhiko Imano: He graduated from Akita University in 1979 and became a research associate in the Department of Electric Engineering at Akita University. He was on leave at Tohoku University as a visiting researcher in 1985-1986. He became a lecturer, an associate professor, and subsequently a professor in 1997 at Akita University. He received his $\mathrm{PhD}$ degree in engineering from Tohoku University in 1993. He is a member of the Institute of Electronics, Information and Communication Engineers, the Japan Acoustical Society of Japan, the Society of Instrument and Control Engineers, the Japanese Society for Non-Destructive Inspection, the Acoustical Society of America, the Institute of Electrical and Electronics Engineers, the Society of Materials Engineering for Resource of Japan, and the Imaging Society of Japan. (imano@gipc.akita-u.ac.jp) 